\title{
Single intraoperative applications of 5-fluorouracil during filtration surgery: early results
}

\author{
Lumina Lanigan, Jörg Stürmer, Karin A Baez, Roger A Hitchings, Peng T Khaw
}

\begin{abstract}
Experimental studies from our laboratory have suggested that single exposures to 5fluorouracil (5-FU) would have a similar effect to subconjunctival injections of 5-FU and a less permanent effect on subconjunctival fibroblasts than mitomycin C (MMC). Thirty four eyes of 33 glaucoma patients with an increased risk of filtration surgery failure were treated with a single intraoperative 5 minute exposure of sclera and subconjunctival tissues to 5-FU, $25 \mathrm{mg} / \mathrm{ml}$. The follow up period ranged from 3-9 months, during which two high risk eyes failed completely and one required topical $\beta$ blockers to control intraocular pressure. No low/moderate risk eyes failed. Single 5 minute intraoperative exposures to 5-FU are convenient, inexpensive, have no significant corneal side effects, and may be a useful adjunctive treatment to optimise the results of glaucoma filtration surgery, particularly in the large group of low/moderate risk patients.

(BrF Ophthalmol 1994; 78: 33-37)
\end{abstract}

The use of postoperative subconjunctival injections of 5-fluorouracil (5-FU) has considerably increased the success rate of filtration surgery in high risk patients. ${ }^{1}$ However, injections are inconvenient and complications include a high incidence of corneal epithelial defects. ${ }^{1}$ The use of intraoperative mitomycin C (MMC) also increases the success rate of glaucoma filtration surgery in high risk patients without significant corneal complications, ${ }^{2-5}$ but the resultant blebs are extremely thin and avascular, and hypotony has been reported ${ }^{2356}$ with documented irreversible maculopathy. ${ }^{6}$

We have recently shown, using in vitro cell culture studies, that single 5 minute exposures to 5-FU and MMC have considerably prolonged effects on the proliferation of human ocular fibroblasts in cell culture. ${ }^{7}$ In an in vivo rabbit model of glaucoma filtration surgery, subconjunctival and scleral fibroblasts grown from treated filtration areas eventually recover within 30 days, following 5 minute treatments with high concentrations of $5-\mathrm{FU}(50 \mathrm{mg} / \mathrm{ml})$ but not after exposure to $M M C$ at a concentration of 0.4 $\mathrm{mg} / \mathrm{ml} .^{8}$ In the same model, the use of single exposures to $5-\mathrm{FU}, 50 \mathrm{mg} / \mathrm{ml}$, significantly prolonged the survival of filtration blebs, but with a less marked effect and thicker blebs compared with $\mathrm{MMC}, 0.2$ and $0.4 \mathrm{mg} / \mathrm{ml} .^{9}$ A preliminary clinical trial using an intraoperative exposure to 5-FU followed by postoperative injections suggested that the addition of intraoperative 5-FU resulted in a greater pressure lowering effect with a reduced need for injections. ${ }^{10}$ Based on these studies, we carried out a pilot study in patients with an increased risk of failing glaucoma filtration surgery. We used a concentration of $5-\mathrm{FU}$ of $25 \mathrm{mg} / \mathrm{ml}$ based on our previous laboratory studies and the fact that it was the highest concentration commercially available in the United Kingdom.

\section{Methods}

Surgery was performed on 34 eyes of 33 patients. Informed consent was obtained from all patients. All patients underwent a modified Cairns-type trabeculectomy without scleral spur removal. A superior rectus bridal suture was placed and a limbal based conjunctival flap was performed without excision of Tenon's capsule. A small surgical sponge was soaked in $5-\mathrm{FU}, 25 \mathrm{mg} / \mathrm{ml}$ (David Bull Laboratories, Warwick, UK) and trimmed to fit between the sclera and the conjunctival flap, taking care not to expose the cut back edge of the conjunctival flap to the 5-FU soaked sponge. A typical sponge measured $6 \mathrm{~mm}$ (length) by $2 \mathrm{~mm}$ (width) by $1 \mathrm{~mm}$ (thickness). The sponge was removed, resoaked, and replaced every minute up to 5 minutes. The area was then rinsed with $20 \mathrm{ml}$ of balanced salt solution over a period of $30-60$ seconds through a Southampton irrigating cannula. Only after this was completed was a third to a half thickness rectangular scleral flap ( $4 \mathrm{~mm}$ by $3 \mathrm{~mm}$ ) dissected and raised to the limbus. This was to minimise any intraocular penetration of 5-FU. A block of tissue measuring approximately $3 \mathrm{~mm}$ by $1 \mathrm{~mm}$ was resected and a peripheral iridectomy performed. The flap was then sutured down with four 10/0 nylon sutures. The back edge of the conjunctival incision was closed in a single layer with 10/0 nylon suture. All patients received postoperative topical dexamethasone metasulphobenzoate $0 \cdot 1 \%$ eye drops four to six times a day and chloramphenicol eye drops four times a day.

Eyes were examined on at least day 1 , week 1 , month $1,3,6$, and 9 and intervals in between if clinically indicated. Postoperative observations and measurements included visual acuity, intraocular pressure (Goldmann applanation tonometry), slit-lamp biomicroscopy, and fundal examination.

\section{Results}

Surgery was performed on 34 eyes of 33 patients with intraocular pressure uncontrolled on maximally tolerated medical therapy. The causes of glaucoma in the eyes are listed in Table 1 and the risk factors for failure ${ }^{11-23}$ are summarised in Table 2 . The group has been divided into a high risk and low/moderate risk group, based on the risk factors. There were 25 male and eight female

Moorfields Eye Hospital,
City Road, London
EC1V 2PD
L Lanigan
J Stürmer
K A Baez
R A Hitchings
P T Khaw
Institute of
Ophthalmology,
Bath Street, London
EC1V 9EL
K A Baez
P T Khaw
Correspondence to:
Mr Peng T Khaw, Glaucoma
Unit, Moorfields Eye
Hospital, London EC1V 2PD.
Accepted for publication
17 August 1993


Table 1 Ocular diagnosis (more than one diagnosis per eye)

\begin{tabular}{lrl}
\hline & & 'Failed' \\
\hline Primary open angle glaucoma & 16 & \\
Rieger's/Axenfeld's & 3 & \\
Pigment dispersion & 3 & \\
Uveitis & 3 & 1 Uncontrolled on maximal medications \\
Congenital glaucoma & 2 & \\
Chronic angle closure glaucoma & 2 & \\
Silicone oil glaucoma & 1 & 1 Controlled on $\beta$ blockers \\
Sturge-Weber & 1 & \\
Trauma & 1 & 1 Uncontrolled on maximal medication \\
Neovascularisation & 1 & \\
\hline
\end{tabular}

patients, of whom 18 were white, 11 AfroCaribbean, and four Asian. Their mean age was $42 \cdot 8$ years (range $2-76$, SD $18 \cdot 3$ ). The patients were using an average of 2.5 (range $1-4$, SD 0.8 ) medications before surgery. The mean highest preoperative intraocular pressure off medication was $41 \cdot 1 \mathrm{~mm} \mathrm{Hg}$ (range 28-70, SD 9.49), falling to $28 \cdot 1 \mathrm{~mm} \mathrm{Hg}$ (range 20-54, SD $8 \cdot 3$ ) on maximal treatment. Mean length of follow up was $5 \cdot 7$ months (range $3-9$, SD $2 \cdot 1$ ), with 19 eyes having a follow up period of 6 months or more.

The mean postoperative intraocular pressure at the last visit was $14.2 \mathrm{~mm} \mathrm{Hg}$ (range $3-40$, SD 6.73 ) representing an average $50 \%$ lowering of intraocular pressure. Thirty one out of 34 eyes are at present not receiving any further pressure lowering treatment. One eye in a 16-year-old patient with previous detachment surgery and silicone oil glaucoma has required treatment with a topical $\beta$ blocker from 1 month after surgery to maintain intraocular pressure at a satisfactory level. Two eyes failed completely, one in a 35-year-old man with uveitis which failed at 1 month after surgery, and another in a 24-year-old man with diabetic neovascular glaucoma who failed at 2 months. All the 'failures' occurred in the high risk group, whereas all the eyes in the low/moderate risk group were unqualified successes (defined as intraocular pressure $<21 \mathrm{~mm} \mathrm{Hg}$ off all medication). With the limited follow up ranging from 3-9 months, this gives an overall unqualified success rate of both groups combined of $91 \%$ at last follow up.

Table 2 Risk factors present in each eye for 'high' and 'low/moderate' risk groups (more than one risk factor per group)

\begin{tabular}{|c|c|c|}
\hline $\begin{array}{l}\text { Risk factors } \\
\text { (more than one per eye) }\end{array}$ & & 'Failed' \\
\hline $\begin{array}{l}\text { High risk group: } \\
\text { Topical medications }>3 \text { years } \\
11-13\end{array}$ & 6 & $\begin{array}{l}1 \text { Controlled on } \beta \text { blockers (patient also }<40 \text { years } \\
\text { of age) }\end{array}$ \\
\hline $\begin{array}{l}\text { Age }<40^{18-22} \\
\text { Afro-Caribbean } \\
\text { Previous failed filter }^{11} \\
\text { Uveitis }^{23}\end{array}$ & $\begin{array}{l}5 \\
3 \\
5 \\
3\end{array}$ & $\begin{array}{l}1 \text { Uncontrolled on maximal medication (patient } \\
\text { also }<40 \text { years) }\end{array}$ \\
\hline Neovascularisation $^{23}$ & 2 & $\begin{array}{l}1 \text { Uncontrolled on maximal medication (patient } \\
\text { also on topical } R x>3 \text { years }\end{array}$ \\
\hline Aphakia/pesudophakia' & 3 & \\
\hline \multicolumn{3}{|c|}{$\begin{array}{l}\text { In the high risk group } 10 / 13(77 \%) \text { eyes achieved the definition of unqualified success (intraocular } \\
\text { pressure }<21 \mathrm{~mm} \mathrm{Hg} \text { off all treatment). } 11 / 13(85 \%) \text { eyes achieved the definition of qualified success } \\
\text { (intraocular pressure }<21 \mathrm{~mm} \mathrm{Hg} \text { on medical treatment) }\end{array}$} \\
\hline $\begin{array}{l}\text { Low/moderate risk group: } \\
\text { Topical medications }>3 \text { years }{ }^{11-13} \\
\text { Age }<40^{12-22} \\
\text { Afro-Caribbean } \\
\end{array}$ & $\begin{array}{r}16 \\
11\end{array}$ & \\
\hline \multicolumn{3}{|c|}{$\begin{array}{l}\text { In the low/moderate risk group } 20 / 20(100 \%) \text { eyes achieved the definition of unqualified success } \\
\text { (intraccular pressure }<21 \mathrm{~mm} \mathrm{Hg} \text { off all treatment) }\end{array}$} \\
\hline \multicolumn{3}{|c|}{$\begin{array}{l}\text { Overall for both groups combined, the unqualified success rate was } 91 \% \text { and the qualified success rate } \\
\text { was } 94 \%\end{array}$} \\
\hline
\end{tabular}

Table 3 Number of eyes with complications

\begin{tabular}{ll}
\hline Punctatecorneal staining & 2 \\
Epithelial loss (5 months post-op pre-existing bullous & \\
keratopathy) & 1 \\
Chronic hypotony (intraocular pressure $<5$ after $3 / 12)$ & 1 \\
Cataract progression & 1 \\
Leak through conjunctival buttonhole (after laser suturelysis) & 1
\end{tabular}

Leak through conjunctival buttonhole (after laser suturelysis)

Complications were seen in six eyes (Table 3). Two developed minor punctate corneal epithelial staining near the bleb. One patient with pre-existing bullous keratopathy developed persistent central corneal epithelial loss 5 months postoperatively, which required a bandage contact lens. Chronic hypotony (defined as an intraocular pressure of less than $5 \mathrm{~mm} \mathrm{Hg}$ after 3 or more months) was seen in one eye, but postoperative visual acuity was within one line of preoperative visual acuity 6 months after surgery. One eye in a 67-year-old AfroCaribbean man had a fall in visual acuity of two lines from $6 / 6$ to $6 / 12$ due to cataract formation. Otherwise visual acuity was within one line or better than preoperative levels in 33 out of 34 eyes (Table 4). Seven eyes had laser suturelysis performed. One eye developed a small temporary leak through a conjunctival wound buttonhole following laser suture lysis performed on the second postoperative day, but this sealed spontaneously within a week without further intervention. The majority of eyes (27/34) had blebs described as cystic, but a diffuse transconjunctival leak of aqueous after fluorescein staining was only noted in one eye with an intraocular pressure of $6 \mathrm{~mm} \mathrm{Hg}$ at the patient's most recent visit.

\section{Discussion}

The overall success rate of $91 \%$ (defined as an intraocular pressure $<21 \mathrm{~mm} \mathrm{Hg}$ of all medications) or $94 \%$ (defined as an intraocular pressure of $<21 \mathrm{~mm} \mathrm{Hg}$ on or off medications) in this mixed group of patients is very encouraging, despite the limitations of a relatively short follow up period. This was a pilot study based on laboratory studies which suggested that a 5 minute exposure to 5 -FU would be as effective as a longer course of subconjunctival injections in inhibiting the proliferation of ocular fibroblasts. ${ }^{74}$ The majority of patients were categorised as having a low or moderately increased chance of failing filtration surgery with risk factors including more than 3 years of topical medication, ${ }^{11-13}$ Afro-Caribbean origin, ${ }^{14-17}$ and age $<40$ years. ${ }^{18-22}$ High risk factors included previous failed filtration surgery, ${ }^{1}$ aphakia, uveitis, and neovascularisation. ${ }^{23}$ The two eyes that completely failed surgery were in patients with uveitis and neovascularisation.

The use of a single 5 minute application of 5-FU during glaucoma filtration surgery to prevent scarring appears to have considerable advantages over regimens involving postoperative injections. Postoperative injections are inconvenient, require multiple hospital visits, and the injections can be uncomfortable for the patient. These problems are not encountered with the single intraoperative application of 5-FU. The incidence of postoperative corneal 


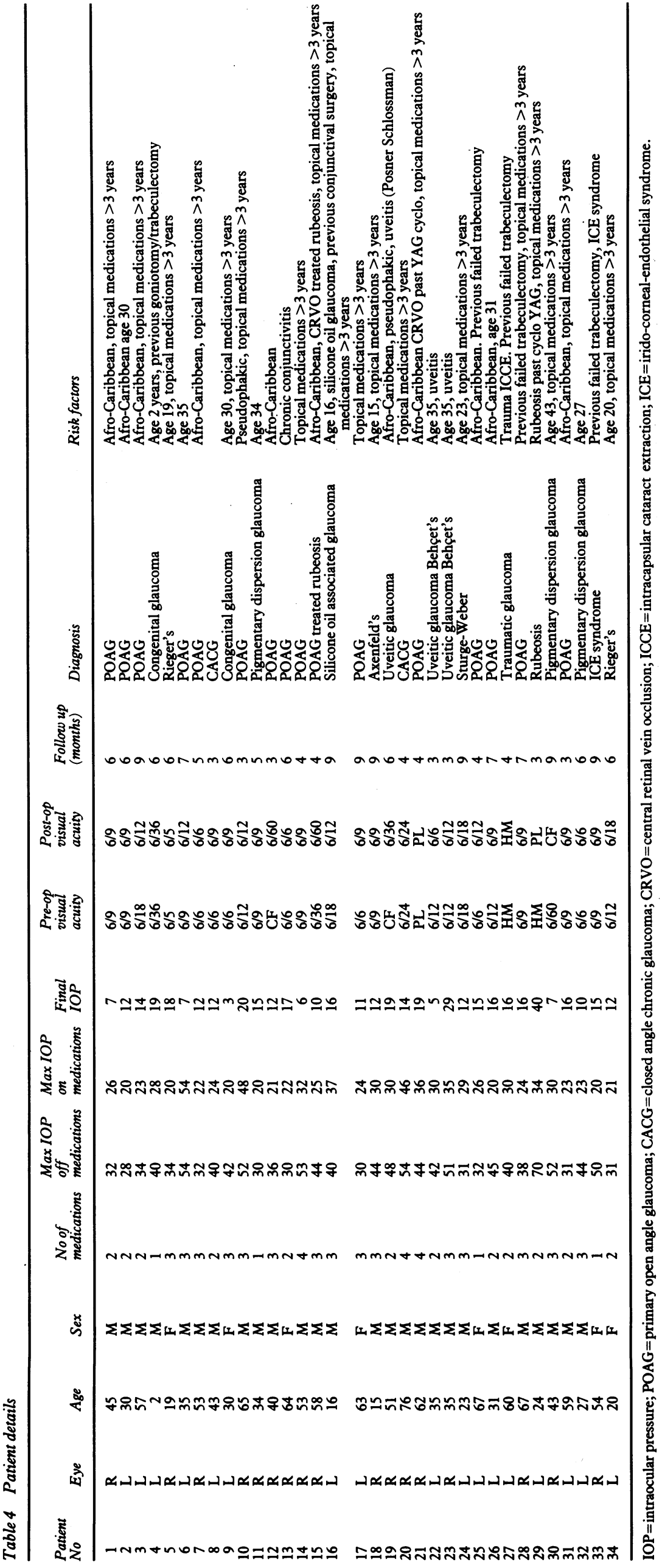


complications with the intraoperative regimen appear to be negligible; in contrast the Fluorouracil Filtering Surgery Study' reported a $64 \%$ incidence of corneal epithelial defects in the first 2 weeks after surgery, and Weinreb ${ }^{25}$ still reported a $29 \%$ incidence of corneal complications using much lower doses of subconjunctival 5-FU. Two eyes in our study had clinically insignificant punctate staining adjacent to the bleb which rapidly resolved.

One other patient with pre-existing traumatic glaucoma and bullous keratopathy developed corneal epithelial loss 4 months after surgery, but this was not thought to be related to the intraoperative use of 5-FU. The reason for this difference in corneal complications may be because the drug is delivered to the inner surface of the conjunctiva and sclera for 5 minutes and then rapidly washed away with the single intraoperative dose regimen. With an injection the 5-FU may leak out into the tear film and be in contact with the corneal epithelium for much longer periods. The low incidence of significant corneal complications seen with intraoperative 5-FU is similar to that seen with the use of intraoperative $M M C .^{2-5}$

We used a volume of $20 \mathrm{ml}$ of balanced salt solution over $30-60$ seconds to wash out the treatment area. The main reason for performing a washout was to prevent any remaining concentrated 5-FU from entering the anterior chamber when the eye was opened. For the size of sponge we used, a maximum of $0.2 \mathrm{ml}$ total volume of $5-\mathrm{FU}(25 \mathrm{mg} / \mathrm{ml})$ is left in the subconjunctival space. This is then diluted at least 100 times by the irrigating fluid which leaves a reasonable margin for safety. However, based on data from Kawase et $a l^{26}$ on the pharmacokinetics of 5 minutes' exposure to MMC (which has similar pharmacokinetics to 5 -FU) followed by $200 \mathrm{ml}$ of saline, and previous data on the pharmacokinetics of subconjunctival $5-\mathrm{FU},{ }^{27}$ it is likely that 'therapeutic' levels of 5-FU are maintained in the tissues for several hours.

None of the patients in our study had a wound leak compared with an incidence as high as $35 \%$ noted in the Fluorouracil Filtering Surgery Study ${ }^{1}$ using postoperative injections. This may be due in part to the fact that the effects of single dose intraoperative treatment with 5-FU are relatively localised to the treated area, ${ }^{8}$ and sparing the cut edge of conjunctiva may leave the wound edge able to heal normally, whereas with injections the drug is distributed throughout the conjunctiva. ${ }^{27}$ Wound leaks have been reported after the use of intraoperative $M M C$ where the back edge of the wound has clearly been exposed to the drug. ${ }^{45}$ However, other studies using lower doses of postoperative 5-FU have reported no wound leaks ${ }^{28}$ as have studies using intraoperative $\mathrm{MMC}^{3}$ However, it was unclear whether or not the back edge of the wound had been exposed to MMC. ${ }^{3}$ One of our patients had a leaking buttonhole following laser suturelysis, but this healed spontaneously. Schwartz and Weiss $^{29}$ reported two cases of conjunctival buttonholes after laser suturelysis in two patients who had received intraoperative $M M C$, but in these cases the leaks persisted considerably longer, something they had not encountered in
175 previous laser suturelyses without MMC. This prolonged inhibition of healing may have been related to the much longer effect of intraoperative MMC compared with 5-FU on fibroblasts in tissues treated with these drugs. ${ }^{8}$

One of the patients in our study has an intraocular pressure of $3 \mathrm{~mm} \mathrm{Hg} 6$ months after surgery with a thin cystic bleb, although there is no overt leak from the bleb. This patient is a 30year-old Asian woman with congenital glaucoma treated with previous goniotomies. Her preoperative visual acuity was $6 / 9$ and was $6 / 12$ at her last postoperative visit without overt maculopathy. Hypotony of $5 \mathrm{~mm} \mathrm{Hg}$ or less has been described following postoperative low dose subconjunctival 5-FU ${ }^{2}$ and intraoperative $M_{M C}{ }^{2-5}$ associated with documented maculopathy. ${ }^{26}$ Although this complication may be expected to be less common with intraoperative 5-FU compared with MMC, based on the relative effects in vivo and in vitro, hypotony is a potentially serious complication that can occur after the use of intraoperative 5-FU. Although no cases of endophthalmitis were seen during this relatively short period of follow up, the fact that the cystic bleb appearance in eyes treated with intraoperative 5-FU is similar to those seen with subconjunctival 5-FU, the long term increased incidence of endophthalmitis ${ }^{30}$ may be similar. However, it may be necessary to have these relatively cystic blebs to achieve the lower intraocular pressures required in many of these patients.

The results of this preliminary pilot study suggest that single 5 minute applications of 5-FU $25 \mathrm{mg} / \mathrm{ml}$ may be a useful adjunctive treatment in patients at risk of failing glaucoma filtration surgery. It has the same advantages as a single intraoperative exposure to MMC which are the removal of the need for postoperative injections, and the virtual absence of any significant corneal complications. A possible advantage of the current intraoperative $5-\mathrm{FU}(25 \mathrm{mg} / \mathrm{ml})$ regimen over current $M M C$ regimens using concentrations of $0.2-0.4 \mathrm{mg} / \mathrm{ml}$, is the fact that the effects on fibroblasts in treated subconjunctival and scleral tissue are much less prolonged, and are reversible ${ }^{8}$ with $5-\mathrm{FU}, 50 \mathrm{mg} / \mathrm{ml}$, compared with MMC, $0.4 \mathrm{mg} / \mathrm{ml}$. Although a direct clinical comparison was not done in this study, it was obvious from personal clinical experience with the use of MMC, that the blebs resulting from intraoperative 5-FU were considerably less avascular than those resulting from the use of intraoperative $M M C$ in humans. It is interesting to speculate whether this may possibly result in a lower long term incidence of endophthalmitis and hypotony with intraoperative 5-FU compared with intraoperative MMC. In a study using an experimental model of glaucoma filtration surgery comparing intraoperative 5-FU, $M M C ~ 0.2 \mathrm{mg} / \mathrm{ml}$, and $M M C ~ 0.4 \mathrm{mg} / \mathrm{ml}$, the only case of endophthalmitis developed in an eye treated with the high concentration of MMC.9

However, in cases where a more prolonged inhibition of local fibroblast proliferation is required, the longer action of MMC may be necessary. It is of interest that of the two eyes that failed after intraoperative 5-FU, one had active neovascularisation and the other had 
active uveitis, two conditions which may be associated with persistent stimulation of the healing response. Only long term randomised prospective trials comparing intraoperative 5-FU, postoperative injections of 5-FU, and intraoperative $M M C$ will clearly establish the relative indications for these different regimens in different patient groups. Finally, although the use of intraoperative or postoperative proliferative agents is at present usually restricted to groups at risk of filtering surgery failure, there is now evidence that the lower intraocular pressures achieved with filtration surgery are associated with an improved visual prognosis. ${ }^{31}$ The use of antiproliferative agents had been shown to result in a lower final intraocular pressure in patients undergoing primary glaucoma filtration surgery. ${ }^{32}{ }^{33}$ Cell culture studies show that the long term inhibition of fibroblast proliferation seen with single dose treatments are clearly titratable. ${ }^{7}$

It is theoretically possible that the single dose treatments with 5-FU could be titrated by altering concentration or surface area treated to achieve a maximal intraocular pressure lowering in all low risk patients, while minimising undesired effects such as thin cystic blebs which may predispose to endophthalmitis and hypotony. Studies are in progress to establish whether or not this is the case.

This study was supported in part by the Wellcome Trust, the International Glaucoma Association, and the Violet M Richards Charity.

The authors state that they have no proprietary interest in any of the products mentioned in this manuscript.

1 The Fluorouracil Filtering Surgery Study Group. Fluorouracil filtering surgery study one-year follow-up. Am F Ophthalmol 1989; 108: 625-35.

2 Chen C-W, Huang H-T, Bair JS, Lee C-C. Trabeculectomy with simultaneous topical application of mitomycin-C in refractory glaucoma. F Ocul Pharmacol 1990; 6: 175-82.

3 Palmer SS. Mitomycin as adjunct chemotherapy with trabeculectomy. Ophthalmology 1991; 98: 317-21.

4 Kitazawa Y, Kawase $K$, Matsushita $H$, Minobe $M$. Trabeculectomy with mitomycin. A comparative study with fluorouracil. Arch Ophthalmol 1991; 109: 1693-8.

5 Skuta GL, Beeson CC, Higginbotham EJ, Lichter PR, Musch DC, Bergstrom TJ, et al. Intraoperative mitomycin versus postoperative 5-fluorouracil in high-risk glaucoma filtering postoperative 5-fluorouracil in high-risk g
surgery. Ophthalmology 1992; 99: 438-44.

6 Jampel HD, Pasquale LR, Dibernardo C. Hypotony maculopathy following trabeculectomy with mitomycin c. Arch Ophthalmol 1992; 110: 1049-50.

7 Khaw PT, Sherwood MB, MacKay SLD, Rossi MK, Schultz GS. Five-minute treatments with fluorouracil, floxuridine, and mitomycin have long-term effects on human Tenon's capsule fibroblasts. Arch Ophthalmol 1992; 110: 1150-4.

8 Khaw PT, Doyle JW, Sherwood MB, Grierson I, Schultz GS, McGorray S. Prolonged localized tissue effects from 5-minute exposures to 5-fluorouracil and mitomycin $\mathrm{C}$. Arch Ophthalmol 1993; 111: 263-7.

9 Khaw PT, Doyle JW, Sherwood MB, Smith MF, McGorray $S$. Effects of intraoperative 5-fluorouracil or mitomycin-c on glaucoma filtration surgery in the rabbit. Ophthalmology 1993; 100: 367-72.

10 Smith MF, Sherwood MB, Doyle JW, Khaw PT. Results of intraoperative 5-fluorouracil supplementation on trabeculectomy for open-angle glaucoma. $A m \quad \mathcal{F}$ Ophthalmol 1992; 114: 737-41.

11 Lavin MJ, Wormald RPL, Migdal CS, Hitchings RA. The influence of prior therapy on the success of trabeculectomy. Arch Ophthalmol 1990; 108: 1543-8.

12 Miller MH, Rice NSC. Trabeculectomy combined with $\beta$ radiation for congenital glaucoma. Br $\mathcal{Y}$ Ophthalmol 1991; 75: 584-90.

13 Longstaff S, Wormald RPL, Mazover A, Hitchings RA. Glaucoma triple procedures: efficacy of intraocular pressure control and visual outcome. Ophthalmic Surg 1990; 21 : 78693.

14 Miller RD, Barber JC. Trabeculectomy in black patients. Ophthalmic Surg 1981; 12: 46-50.

15 Berson D, Landau L, Zauberman H, Blumenthal M. Filtering operations in Africans. Am $\mathcal{F}$ Ophthalmol 1969; 67: 395-8.

16 Iliff CE. Surgical control of glaucoma in the Negro. Am F Ophthalmol 1944; 27: 731-8.

17 Merritt JC. Filtering procedures in American blacks. Ophthalmic Surg 1980; 11: 91-4.

18 Inaba $Z$. Long term results of trabeculectomy in the Japanese: an analysis by life-table method. $\mathcal{F p n} \mathcal{f}$ Ophthalmol 1982; 26: 361-73.

19 Heuer DK, Parrish RK II, Gressel MG, Anderson DR, Hodapp E, Palmberg PF. Trabeculectomy in the aphakic eyes. Ophthalmology 1984; 91: 1045-51.

20 Stewart RH, Kimbrough RL, Bach H, Allbright $M$. Trabeculectomy and modifications of trabeculectomy. Ophthalmic Surg 1979; 10: 76-80.

21 Schwartz AL, Anderson DR. Trabecular surgery. Arch Ophthalmol 1974; 92: 134-8.

22 Sturmer J, Broadway DC, Hitchings RA. Young patient trabeculectomy: assessment of risk factors for failure. trabeculectomy: assessment of
Ophthalmology 1993; 100: 928-39.

23 Heuer DK, Parrish RKII, Gressel MG, Hodapp E, Desjardins DC, Skuta GL, et al. 5-Fluorouracil and glaucoma filtering surgery. III. Intermediate follow-up of a pilot study. Ophthalmology 1986; 93: 1537-46.

24 Khaw PT, Ward S, Porter A, Grierson I, Hitchings RA, Rice NSC. The long-term effects of 5-fluorouracil and sodium butyrate on human Tenon's fibroblasts. Invest Ophthalmol Vis Sci 1992; 33: 2043-52.

25 Weinreb RN. Adjusting the dose of 5-fluorouracil after filtration surgery to minimize side effects. Ophthalmology 1987; 94: 564-70.

26 Kawase K, Matsushita H, Yamamoto T, Kitazawa Y. Mitomycin concentration in rabbit and human ocular tissues after topical administration. Ophthalmology 1992; 99: 203-7.

27 Kondo M, Araie M. Concentration change of fluorouracil in the external segment of the eye after subconjunctival injection. Arch Ophthalmol 1988; 106: 1718-21.

28 Whiteside-Michel J, Liebmann JM, Ritch R. Initial 5-fluorouracil trabeculectomy in young patients. Ophthalmology 1992; 99: 7-13.

29 Schwartz AL, Weiss HS. Bleb leak with hypotony after laser suture lysis and trabeculectomy with mitomycin C. Arch Ophthalmol 1992; 110: 1049.

30 Wolner B, Liebmann JM, Sassani JW, Ritch R, Speaker M, Marmor M. Late bleb-related endophthalmitis after Mabeculectomy with adjunctive 5-fluorouracil. trabeculectomy with adjun

31 Jay JL, Allan D. The benefit of early trabeculectomy versus conventional management in primary open angle glaucoma relative to severity of disease. Eye 1989; 3: 528-35.

32 Liebmann JM, Ritch R, Marmor M, Nunez J, Warner B. Initial 5-fluorouracil trabeculectomy in uncomplicated glaucoma. Ophthalmology 1991; 98: 1036-41.

33 Ophir A, Ticho U. A randomized study of trabeculectomy and subconjunctival administration of fluorouracil in primary glaucomas. Arch Ophthalmol 1992; 110: 1072-5. 